ESAIM: PROCEEDINGS AND SURVEYS, December 2016, Vol. 55, p. 41-60

Emmanuel FRÉNOD, Emmanuel MAITRE, Antoine ROUSSEAU, Stéphanie SALMON and Marcela SZOPOS Editors

\title{
STUDY OF A DEPRESSURISATION PROCESS AT LOW MACH NUMBER IN A NUCLEAR REACTOR CORE
}

\author{
Andrea Bondesan ${ }^{1}$, Stéphane Dellacherie ${ }^{2}$, Hélène Hivert ${ }^{3}$, \\ Jonathan Jung ${ }^{4}$, VAnessa Lleras ${ }^{5}$, Colin Mietka ${ }^{6}$ And Yohan Penel ${ }^{7}$
}

\begin{abstract}
This paper deals with the numerical treatment of two additional terms in the LMNC-system modelling the coolant in a nuclear reactor core. The latter model was derived and studied by the authors in previous publications. On the one hand, we investigate the influence of the thermal conduction upon steady analytical solutions and upon numerical strategies designed in dimensions 1 and 2. On the other hand, we consider a time-varying thermodynamic pressure that enables to simulate a larger variety of physical situations. Taking into account the resulting terms in the equations lead us to adapt numerical methods to ensure accuracy. In particular, we use an adaptative mesh refinement procedure whose level of refinement depends on the phase.
\end{abstract}

Résumé. Le système d'équations LMNC, étudié précédemment par les auteurs, permet de modéliser l'eau dans le circuit primaire d'un réacteur nucléaire. Dans ce papier, nous nous intéressons à l'enrichissement du modèle par la prise en compte de deux phénomènes : d'une part la diffusion thermique qui influe sur les états stationnaires et sur les schémas numériques mis en œuvre en dimensions 1 et 2 , et d'autre part la dépendance en temps de la pression thermodynamique afin de pouvoir simuler des situations physiques plus variées. Les nouveaux termes intervenant dans les équations nécessitent d'adapter les outils numériques afin de maintenir la précision des résultats. En particulier, nous utilisons une procédure de raffinement de maillage adaptatif dont le niveau de raffinement dépend du type de phase.

\footnotetext{
1 MAP5 UMR CNRS 8145 - Sorbonne Paris Cité - Université Paris Descartes 75270 Paris Cedex 6, France \& e-mail: andrea.bondesan@parisdescartes.fr

2 DEN/DANS/DM2S/STMF - Commissariat à l'Énergie Atomique et aux Énergies Alternatives - Saclay, 91191 Gif-sur-Yvette, France and Département de Génie Mécanique, École Polytechnique de Montréal, 2900 Boulevard Edouard-Montpetit, Montréal, QC H3T 1J4, Canada \& e-mail: stephane.dellacherie@cea.fr

${ }^{3}$ IRMAR - Institut de Recherche Mathématique de Rennes and IPSO (Inria Rennes - Bretagne atlantique and IRMAR) \& e-mail: helene.hivert@inria.fr

${ }^{4}$ LMA-IPRA UMR CNRS 5142 - Université de Pau et des Pays de l'Adour, 64013 Pau, France, and CAGIRE (Inria Bordeaux Sud Ouest) \& e-mail: jonathan.jung@univ-pau.fr

${ }^{5}$ IMAG UMR CNRS 5149 - Université de Montpellier, CC051, 34095 Montpellier cedex 5

\& e-mail: vanessa.lleras@umontpellier.fr

${ }^{6}$ ICJ UMR CNRS 5208, Université Lyon 1, 69622 Villeurbanne cedex \& e-mail: mietka@math.univ-lyon1.fr

7 ANGE (CEREMA and Inria Paris) and LJLL UMR CNRS 7598 - Sorbonne Universités, UPMC Univ. Paris 06, 75005 Paris, France \& e-mail: yohan.penel@cerema.fr
}

(C) EDP Sciences, SMAI 2017 


\section{INTRODUCTION}

For the purpose of studying thermodynamic and kinematic properties of the coolant fluid in the primary circuit of a pressurised water reactor (PWR), the low Mach nuclear core model (LMNC) was introduced in [4]. As the Navier-Stokes equations modelling the behaviour of a compressible fluid raise several theoretical and numerical issues, a simplification process was applied. The incompressible model was not under consideration since it does not account for high thermal/pressure variations. That is why an intermediate system was derived by means of an asymptotic expansion with respect to the Mach number $[15,16,18]$ whose order of magnitude is reportedly small in the situations of interest.

Due to the decoupling of the pressure field into thermodynamic/dynamic pressure variables, the mathematical structure of the resulting LMNC model is easier to deal with, whether it be concerning the analysis (derivation of analytical solutions in dimension 1) or for numerical purposes (coupling of robust numerical methods). The major advantage of this approach is that the thermodynamic pressure involved in the equation of state (EOS) does not depend on space variables. In this setting, it is prescribed by boundary conditions. In other cases, it can be seen as the solution of an ODE [10].

In previous works, the data was chosen so that the thermodynamic pressure is constant. In dimension 1, the authors computed transient analytical solutions without phase transition [2] and with phase transition [3]. A numerical scheme based on the method of characteristics was proposed [3]. The extension to dimension 2 was carried out in $[2,5]$ by means of the finite element software FrEEFEM $++[14]$ still coupled to the method of characteristics [21]. All these results were obtained modelling the thermodynamic properties of water with the Stiffened Gas EOS whose parameters are tuned separately for the liquid phase and the vapour phase. To improve the physical accuracy of the results in the range of pressures and temperatures at stake, the analytical EOS was replaced in [6] by fitting polynomials using experimental values extracted from [17].

The present work consists in enriching the model by taking into account two additional phenomena. On the one hand, we investigate the case of a time-varying thermodynamic pressure which enables to deal with more general situations like depressurisation processes for safety evaluations. This induces new terms in the equations but it does not raise additional issues in the design of the numerical scheme. On the other hand, we aim at pointing out the low influence of thermal diffusion effects on the overall behaviour of the fluid. The resulting additional terms in the formulation change the mathematical structure of the equation and requires substantial modifications of the numerical schemes.

The LmNC model reads

$$
\left\{\begin{array}{l}
\nabla \cdot \boldsymbol{u}=-\frac{p_{*}^{\prime}(t)}{\rho\left(h, p_{*}(t)\right) c^{2}\left(h, p_{*}(t)\right)}+\frac{\beta\left(h, p_{*}(t)\right)}{p_{*}(t)}\left[\nabla \cdot\left(\Lambda\left(h, p_{*}(t)\right) \nabla h\right)+\Phi\right], \\
\rho\left(h, p_{*}(t)\right) \times\left[\partial_{t} h+\boldsymbol{u} \cdot \nabla h\right]=\nabla \cdot\left[\Lambda\left(h, p_{*}(t)\right) \nabla h\right]+\Phi+p_{*}^{\prime}(t), \\
\rho\left(h, p_{*}(t)\right) \times\left[\partial_{t} \boldsymbol{u}+(\boldsymbol{u} \cdot \nabla) \boldsymbol{u}\right]-\nabla \cdot \sigma(\boldsymbol{u})+\nabla \bar{p}=\rho\left(h, p_{*}(t)\right) \boldsymbol{g},
\end{array}\right.
$$

for $(t, \boldsymbol{x}) \in \mathbb{R}^{+} \times \Omega_{d}{ }^{1}$ Initial conditions are $h(0, \boldsymbol{x})=h_{0}(\boldsymbol{x}), \boldsymbol{u}(0, \boldsymbol{x})=\boldsymbol{u}_{0}(\boldsymbol{x})$ and $\bar{p}(0, \boldsymbol{x})=0$.

The unknowns are the enthalpy $h$, the velocity field $\boldsymbol{u}$ and the dynamic pressure $\bar{p}$. Data are the thermodynamic pressure $t \mapsto p_{*}(t)$, the power density of the core $(t, \boldsymbol{x}) \mapsto \Phi(t, \boldsymbol{x})$ and the gravity

\footnotetext{
${ }^{1} \Omega_{1}=\left[0, L_{y}\right]$ and $\Omega_{2}=\left[0, L_{x}\right] \times\left[0, L_{y}\right]$
} 


\begin{tabular}{|c|c|c|c|c|}
\hline & $\beta(h, p)$ & $q(h, p)$ & $T(h, p)$ & $\Lambda(h, p)$ \\
\hline \multirow{2}{*}{$h \leq h_{\ell}^{s}(p)$} & $\gamma_{l}-1 \quad p$ & $\alpha$ & $\underline{h-q_{l}}$ & $\underline{\lambda_{l}}$ \\
\hline & $\gamma_{\ell} \quad p+\pi_{\ell}$ & $9 l$ & $c_{p \ell}$ & $c_{p_{\ell}}$ \\
\hline$h_{\ell}^{s}(p)<h<h_{g}^{s}(p)$ & $p \cdot \frac{\frac{1}{\rho_{q}^{s}}-\frac{1}{\rho_{\ell}^{s}}}{h_{q}^{s}-h_{\ell}^{s}}(p)$ & $\frac{\rho_{g}^{s} h_{q}^{s}-\rho_{\ell}^{s} h_{\ell}^{s}}{\rho_{g}^{s}-\rho_{\ell}^{s}}(p)$ & $T^{s}(p)$ & 0 \\
\hline$h \geq h_{g}^{s}(p)$ & $\frac{\gamma_{g}-1}{\gamma_{g}} \frac{p}{p+\pi_{q}}$ & $q_{q}$ & $\frac{h-q_{q}}{c_{p}}$ & $\frac{\lambda_{q}}{c_{p}}$ \\
\hline
\end{tabular}

TABLE 1. Stiffened Gas law and expression of $\Lambda$ for the three phases

field $\boldsymbol{g}$. The Cauchy stress tensor $\sigma$ is classically expressed involving the Lamé viscosity coefficients:

$$
\sigma(\boldsymbol{u})=\mu_{0}\left(\nabla \boldsymbol{u}+(\nabla \boldsymbol{u})^{T}\right)+\eta_{0}(\nabla \cdot \boldsymbol{u}) \mathcal{I}_{d}
$$

where $\mu_{0}$ and $\eta_{0}$ are constant. Moreover, we assume that $\eta_{0}=-\frac{2}{3} \mu_{0}$ (Stokes hypothesis). Other variables such as density $\rho$, speed of sound $c$ or compressibility coefficient $\beta \stackrel{\text { def }}{=}-\frac{p}{\rho^{2}} \frac{\partial \rho}{\partial h}$, are evaluated by means of the EOS or empirical laws as functions of $h$ and $p_{*}$ with separate expressions depending on the phases (liquid, mixture, gas). The reader is referred to [6] for more details about the modelling of the mixture phase and the computation of variables at saturation. Our modelling is enthalpy-oriented: the values of the enthalpy determine the related phase and all thermodynamic variables are deduced from $h$. Denoting by $p \mapsto h_{\ell}^{s}(p)$ and $p \mapsto h_{g}^{s}(p)$ the enthalpies at saturation in the liquid and in the vapour, we have

$$
\zeta(h, p)= \begin{cases}\zeta_{\mathfrak{l}}(h, p), & \text { if } h \leq h_{\mathfrak{l}}^{s}(p), \\ \zeta_{\mathfrak{m}}(h, p), & \text { if } h_{\mathfrak{l}}^{s}(p)<h<h_{\mathfrak{g}}^{s}(p), \\ \zeta_{\mathfrak{g}}(h, p), & \text { if } h \geq h_{\mathfrak{g}}^{s}(p),\end{cases}
$$

for $\zeta \in\{\rho, \beta, \Lambda, T, c\}$. $\zeta_{\boldsymbol{l}}$ and $\zeta_{\boldsymbol{g}}$ are expressions of EOS or empirical laws in the liquid and vapour phases. $\zeta_{m}$ comes from modelling choices (see [6]). In particular, coefficient $\Lambda$ involved in System (1) comes from consequences of classical laws of thermodynamics that state

$$
\Lambda(h, p) \nabla h=\lambda(h, p) \nabla T(h, p), \quad \text { with } \quad \Lambda(h, p)= \begin{cases}\Lambda_{\mathfrak{l}}(h, p) \stackrel{\text { def }}{=} \frac{\lambda_{\mathfrak{l}}(h, p)}{c_{p \mathfrak{l}}(h, p)}, & \text { if } h \leq h_{\mathfrak{l}}^{s}(p), \\ \Lambda_{\mathfrak{m}}(h, p) \equiv 0, & \text { if } h_{\mathfrak{l}}^{s}(p)<h<h_{\mathfrak{g}}^{s}(p), \\ \Lambda_{\mathfrak{g}}(h, p) \stackrel{\text { def }}{=} \frac{\lambda_{\mathfrak{g}}(h, p)}{c_{p_{\mathfrak{g}}}(h, p)}, & \text { if } h \geq h_{\mathfrak{g}}^{s}(p),\end{cases}
$$

where $\lambda$ is the thermal conductivity and $c_{p}$ the heat capacity at constant pressure. The value in the mixture is set to 0 as the temperature is constant within the saturation dome.

As our goal is here to derive relevant numerical methods and to assess them, the sequel is restricted to the Stiffened Gas law for the sake of clarity. Indeed, it is then possible to exhibit analytical steady solutions (Prop. 1) which will be compared to numerical solutions. It can be then applied to tabulated laws without restriction. 


\begin{tabular}{ccc}
\hline Location & Enthalpy & Velocity/pressure \\
\hline $\begin{array}{c}\text { Bottom } \\
(y=0)\end{array}$ & $h=h_{e}$ & $\rho\left(h_{e}, p_{*}\right) \boldsymbol{u}=\left(0, D_{e}\right)$ \\
\hline $\begin{array}{c}\text { Lateral } \\
\left(x \in\left\{0, L_{x}\right\}\right)\end{array}$ & $\Lambda\left(h, p_{*}\right) \nabla h \cdot \boldsymbol{n}=0$ & $\begin{array}{c}\boldsymbol{u} \cdot \boldsymbol{n}=0 \\
\sigma(\boldsymbol{u}) \boldsymbol{n} \cdot \boldsymbol{\tau}=0\end{array}$ \\
\hline $\begin{array}{c}\text { Top } \\
\left(y=L_{y}\right)\end{array}$ & $\Lambda\left(h, p_{*}\right) \nabla h \cdot \boldsymbol{n}=0$ & $\sigma(\boldsymbol{u}) \boldsymbol{n}-\bar{p} \boldsymbol{n}=\mathbf{0}$ \\
\hline
\end{tabular}

TABLE 2. Boundary conditions associated to the LMNC model

The Stiffened Gas law [3] corresponds to the following expression for the density

$$
\rho(h, p)=\frac{p / \beta(h, p)}{h-q(h, p)} .
$$

where $\beta$ and $q$ are given in Table 1 . Values of parameters $\gamma_{\kappa}, \pi_{\kappa}, q_{\kappa}$ and $c_{p_{\kappa}}, \kappa \in\{\boldsymbol{\ell}, \boldsymbol{g}\}$, for $p_{*}=155$ bar are given in [3, Table 1]. The expression of the speed of sound can be found in [3, Appendix B].

Boundary conditions are prescribed by Table 2 . Notice that when $\Lambda$ vanishes, no condition is imposed upon $h$ except at the bottom $y=0$ where the flow goes inward. Hence under this condition the model is compatible with the degeneracy of the thermal conductivity.

We finally assume:

- the input rate $D_{e}$ is non-negative;

- the thermodynamic pressure $p_{*}$ remains non-negative;

- $h_{e}$ is such that $\rho_{e}$ computed thanks to (3) exists and is positive; moreover, $h_{e}$ corresponds to a liquid phase;

- initial data $\left(h_{0}, \boldsymbol{u}_{0}\right)$ are well prepared, i.e. they satisfy Equation (1a).

The discontinuity of $\beta$ and $\Lambda$ at phase change - see Table 1 and (2) - requires some remarks. The fact that $\Lambda$ vanishes in the mixture phase changes the nature of Eq. (1b): it is parabolic (advection-diffusion) in the pure phases (liquid and vapour) but turns hyperbolic (advection) in the mixture phase. This is a coupling of models of different natures with a moving interface (level sets ${ }^{2} \Gamma_{\kappa}$ are unknown). It is a hard mathematical problem which will not be studied from a theoretical point of view (regularity, well-posedness, ...) in this paper. Likewise, we have to specify what kind of solutions to System (1) we are investigating. From now on, this work is restricted to solutions $(h, \boldsymbol{u}, \bar{p})$ of $(1)$ such that:

(1) $(h, \boldsymbol{u}, \bar{p})$ is a strong solution of System (1) in each phase domain ${ }^{3} \omega_{\kappa}(t)$;

$$
\begin{aligned}
& { }^{2} \Gamma_{\ell}(t):=\left\{\boldsymbol{x} \in \Omega_{d} \mid h(t, \boldsymbol{x})=h_{\ell}^{s}\right\}, \Gamma_{\boldsymbol{g}}(t):=\left\{\boldsymbol{x} \in \Omega_{d} \mid h(t, \boldsymbol{x})=h_{q}^{s}\right\} . \\
& { }^{3} \omega_{\ell}(t):=\left\{\boldsymbol{x} \in \Omega_{d} \mid h(t, \boldsymbol{x})<h_{\ell}^{s}\right\}, \omega_{\mathfrak{m}}(t):=\left\{\boldsymbol{x} \in \Omega_{d} \mid h_{\ell}^{s}<h(t, \boldsymbol{x})<h_{q}^{s}\right\}, \omega_{\boldsymbol{g}}(t):=\left\{\boldsymbol{x} \in \Omega_{d} \mid h(t, \boldsymbol{x})>h_{q}^{s}\right\} .
\end{aligned}
$$


(2) $(h, \boldsymbol{u}, \bar{p})$ satisfies jump conditions at each phase interface $\Gamma_{\kappa}(t)$ which ensure the continuity of lagrangian ${ }^{4}$ fluxes:

$$
\begin{gathered}
\llbracket \Lambda\left(h, p_{*}\right) \nabla h \rrbracket \cdot \boldsymbol{n}=0, \\
\llbracket \sigma(\boldsymbol{u})-\bar{p} \mathcal{I}_{d} \rrbracket \boldsymbol{n}=0 ;
\end{gathered}
$$

(3) $h$ satisfies the continuity condition

$$
\llbracket h \rrbracket=0
$$

across interfaces $\Gamma_{\kappa}$ such that $\boldsymbol{u} \cdot \boldsymbol{n}_{\text {hyp }}>0$ where $\boldsymbol{n}_{\text {hyp }}$ is the inward normal vector to the mixture domain $\omega_{m}$ where (1b) degenerates to a hyperbolic equation. ${ }^{5}$

Transmission conditions (4) are those associated to the conservative ${ }^{6}$ formulation of System (1), i.e.

$$
\left\{\begin{array}{l}
\partial_{t} \rho+\nabla \cdot(\rho \boldsymbol{u})=0, \\
\partial_{t}(\rho h)+\nabla \cdot(\rho h \boldsymbol{u}-\Lambda \nabla h)=\Phi+p_{*}^{\prime}(t), \\
\partial_{t}(\rho \boldsymbol{u})+\nabla \cdot\left(\rho \boldsymbol{u} \otimes \boldsymbol{u}-\sigma(\boldsymbol{u})+\bar{p} \mathcal{I}_{d}\right)=\rho \boldsymbol{g}
\end{array}\right.
$$

This kind of coupling methods is investigated for instance in $[9,12]$. As stated above, the theoretical study of transmission conditions is not the goal of the present work.

\section{Study of the 1-Dimensional CASE}

In dimension 1, the system has a particular structure as the momentum equation (1c) decouples from the others. Hence in this section we only focus on the subsystem

$$
\left\{\begin{array}{l}
\partial_{y} v=-\frac{p_{*}^{\prime}(t)}{\rho\left(h, p_{*}(t)\right) c^{2}\left(h, p_{*}(t)\right)}+\frac{\beta\left(h, p_{*}(t)\right)}{p_{*}(t)}\left[\partial_{y}\left(\Lambda\left(h, p_{*}(t)\right) \partial_{y} h\right)+\Phi\right], \\
\rho\left(h, p_{*}(t)\right) \times\left[\partial_{t} h+v \cdot \partial_{y} h\right]=\partial_{y}\left[\Lambda\left(h, p_{*}(t)\right) \partial_{y} h\right]+\Phi+p_{*}^{\prime}(t) .
\end{array}\right.
$$

The dynamic pressure $\bar{p}$ is then recovered by a direct integration of (1c). When $\Lambda \equiv 0$, the Stiffened Gas law implies that $\rho c^{2}$ and $\beta$ are piecewise constant and (6a) decouples from (6b): this no longer holds as soon as one uses another EOS or when $\Lambda \neq 0$.

\subsection{Steady solutions}

Transient 1D solutions can be found in [3,13] for constant $p_{*}$ and $\lambda=0$, and in [20] for perfect gas, time-varying $p_{*}$ and $\lambda=0$. When such solutions cannot be derived, one can obtain steady solutions in order to have explicit elements to assess numerical methods. In the steady case, it comes down to focusing on the influence of thermal conduction as $p_{*}$ is necessarily a constant.

\footnotetext{
${ }^{4}$ The choice of lagrangian fluxes rather than eulerian fluxes is due to the numerical schemes applied in the sequel, namely the method of characteristics.

${ }^{5}$ This transmission condition only holds when the fluid moves from the parabolic area to the hyperbolic domain.

${ }^{6}$ The term "conservative" refers to the differential operators and not to the equation itself which is obvisouly not conservative due to source terms.
} 
For the sake of simplicity, we assume that the power density is constant to $\Phi_{0}>0$ and we set

$$
\mathcal{H}(z)=h_{\ell}^{s}-h_{e}-\frac{\Phi_{0}}{D_{e}} z+\frac{\Phi_{0} \kappa_{\ell}}{D_{e}}\left(1-e^{-z / \kappa_{\ell}}\right), \quad \text { where } \kappa_{\ell} \stackrel{\text { def }}{=} \frac{\Lambda_{\ell}}{D_{e}} .
$$

$\mathcal{H}$ is monotone-decreasing and by assumption, $\mathcal{H}(0)>0$.

It is possible to provide a continuous steady solution of the problem as long as no vapour appears:

\section{Proposition 1.}

(a) If $\mathcal{H}\left(L_{y}\right)>0$, there exists a unique steady solution corresponding to a pure liquid configuration and given by

$$
h_{\infty}(y)=h_{e}+\frac{\Phi_{0}}{D_{e}} y-\frac{\Phi_{0} \kappa_{\ell}}{D_{e}} e^{-L_{y} / \kappa_{\ell}}\left(e^{y / \kappa_{\ell}}-1\right) .
$$

(b) Let us assume that $\mathcal{H}\left(L_{y}\right) \leq 0$ and that $\mathcal{H}\left(L_{y}\right)>\frac{\Phi_{0} \kappa_{\ell}}{D_{e}}\left(e^{-y_{\ell}^{s} / \kappa_{\ell}}-e^{-L_{y} / \kappa_{\ell}}\right)-\left(h_{q}^{s}-h_{\ell}^{s}\right)$ where $y_{\ell}^{s}$ is the unique solution in $\left[0, L_{y}\right]$ of $\mathcal{H}\left(y_{\ell}^{s}\right)=0$. Then there exists a unique steady solution satisfying (4-5) corresponding to a liquid-mixture configuration and given by

$$
h_{\infty}(y)= \begin{cases}h_{e}+\frac{\Phi_{0}}{D_{e}} y-\frac{\Phi_{0} \kappa_{\ell}}{D_{e}} e^{-y_{\ell}^{s} / \kappa_{\ell}}\left(e^{y / \kappa_{\ell}}-1\right), & \text { if } y \in\left(0, y_{\ell}^{s}\right), \\ h_{\ell}^{s}+\frac{\Phi_{0}}{D_{e}}\left(y-y_{\ell}^{s}\right), & \text { if } y \in\left(y_{\ell}^{s}, L_{y}\right) .\end{cases}
$$

Proof. Let us first remark that combining the steady counterparts of $(6 \mathrm{a}-6 \mathrm{~b})$ leads to $\partial_{y}(\rho v)=0 .^{7}$ Hence $\rho v=D_{e}$.

(a) Assume that only liquid is present in the steady state. Then Equation (6b) reads

$$
\partial_{y y}^{2} h-\frac{1}{\kappa_{\ell}} \partial_{y} h=-\frac{\Phi_{0}}{D_{e} \kappa_{\ell}}
$$

Solving this 1st-order ODE for $\partial_{y} h$ and applying the boundary condition on the top yields

$$
\partial_{y} h(y)=\frac{\Phi_{0}}{D_{e}}\left(1-e^{\left(y-L_{y}\right) / \kappa_{\ell}}\right)
$$

We deduce that $h$ is monotone-increasing as well as the expression given in Prop. 1(a). The preliminary assumption (reactor filled with liquid) is valid as long as $h\left(L_{y}\right)<h_{\ell}^{s}$ which is equivalent to $\mathcal{H}\left(L_{y}\right)>0$.

(b) If $\mathcal{H}\left(L_{y}\right) \leq 0$, there cannot be only liquid in the core. We are thus looking for a weak solution of the steady LMNC model which corresponds to a liquid phase over $\left(0, y_{\ell}^{s}\right)$ (for some $y_{\ell}^{s}$ to be determined) and to a mixture phase over $\left(y_{\ell}^{s}, L_{y}\right)$. We recover the test case dealt in [12] except that the interface is not fixed a priori and depends on the solution itself. Transmission conditions (4-5) thus correspond to the variational approach in the aforementioned paper. As $v=D_{e} / \rho>0$, (5) is taken into account. Jump condition (4a) reads

$$
\left(\Lambda\left(h, p_{*}\right) \partial_{y} h\right)_{y_{\ell}^{s-}}=0
$$

${ }^{7}$ We recall that $\beta \stackrel{\text { def }}{=}-\frac{p}{\rho^{2}} \frac{\partial \rho}{\partial h}$. 
Notice that the continuity of $h$ at $y=y_{\ell}^{s}$ implies the continuity of $\rho\left(h, p_{*}\right)$ and then the continuity of $v=D_{e} / \rho\left(h, p_{*}\right)$.

We integrate ODE $(7)$ over $\left(0, y_{\ell}^{s}\right)$ taking boundary condition (8) into account. This leads to

$$
h(y)=h_{e}+\frac{\Phi_{0}}{D_{e}}\left(y-\kappa_{\ell} e^{-y_{\ell}^{s} / \kappa_{\ell}}\left(e^{y / \kappa_{\ell}}-1\right)\right) .
$$

$y_{\ell}^{s}$ is determined by solving the equation $h\left(y_{\ell}^{s}\right)=h_{\ell}^{s}$ which is equivalent to $\mathcal{H}\left(y_{\ell}^{s}\right)=0$, whose root is unique. As for the domain $\left(y_{\ell}^{s}, L_{y}\right)$, we trivially obtain the expression for $h$ as $\Lambda_{m}=0$. This holds as long as $h_{q}^{s}$ is not reached. If so, it is necessary at the boundary $y=L_{y}$ first. The restriction thus reads $h\left(L_{y}\right)=h_{\ell}^{s}+\frac{\Phi_{0}}{D_{e}}\left(L_{y}-y_{\ell}^{s}\right)<h_{\mathfrak{g}}^{s}$ which can be turned into the equivalent inequality in terms of $\mathcal{H}$ given in the statement of Prop. 1(b).

Remark. Similar results with a third phase could be derived but discontinuities occur: indeed (5) is not taken into account anymore and the continuity of the flux ensured by (4a) does not guarantee the continuity of $h$. This will be investigated in future works from theoretical and numerical points of view.

\subsection{Numerical scheme}

The numerical strategy implemented in the present work consists in adapting the numerical method of characteristics (MOC) designed in $[3,19]$ to decouple the two equations in (6). As the MOC scheme is unconditionally stable, the point is here to take into account the diffusion term in Equation (6b) without imposing a restrictive (parabolic) stability condition. That is why an implicit version is considered. Equation (6a) is directly integrated in a second step.

We consider a homogeneous cartesian grid $\left(y_{i}=i \Delta y\right)_{0 \leq i \leq N_{y}}$ where $\Delta y=\frac{L_{y}}{N_{y}}$ for some integer $N_{y} \geq 1$ and a uniform time discretisation $\left(t^{n}=n \Delta t\right)_{n \geq 0}$ for some time step $\Delta t>0$.

More precisely, to solve Equation (6b), we introduce the characteristic field $\tau \mapsto \chi\left(\tau ; t^{n+1}, y_{i}\right)$ as the solution of the ODE

$$
\left\{\begin{array}{l}
\frac{\mathrm{d}}{\mathrm{d} \tau} \chi\left(\tau ; t^{n+1}, y_{i}\right)=v\left(\tau, \chi\left(\tau ; t^{n+1}, y_{i}\right)\right), \quad \tau \leq t^{n+1} \\
\chi\left(t^{n+1} ; t^{n+1}, y_{i}\right)=y_{i}
\end{array}\right.
$$

so that Equation $(6 \mathrm{~b})$ reads

$$
\frac{\mathrm{d}}{\mathrm{d} \tau} h\left(\tau, \chi\left(\tau ; t^{n+1}, y_{i}\right)\right)=\frac{p_{*}^{\prime}(\tau)+\Phi\left(\tau, \chi\left(\tau ; t^{n+1}, y_{i}\right)\right)}{\rho\left(h\left(\tau, \chi\left(\tau ; t^{n+1}, y_{i}\right)\right), p_{*}(\tau)\right)}+\left[\frac{\partial_{y}\left(\Lambda\left(h, p_{*}\right) \partial_{y} h\right)}{\rho\left(h, p_{*}\right)}\right]\left(\tau, \chi\left(\tau ; t^{n+1}, y_{i}\right)\right)
$$

Given the numerical solution $\left(h_{i}^{n}, v_{i}^{n}\right)$ at time $t^{n}$, solutions of Equation (9) can be approximated by means of a numerical scheme of 1st or 2nd order (see [19] for more details) so as to compute the numerical foot of the characteristic curve defined by

$$
\xi_{i}^{n} \approx \chi\left(t^{n} ; t^{n+1}, y_{i}\right)
$$

As $\xi_{i}^{n}$ may not be located at a mesh node, an interpolation process is required to approximate $h\left(t^{n}, \xi_{i}^{n}\right)$. A 3rd-order accurate procedure ensuring the maximum principle is described in [3]. Denoting by $\hat{h}_{i}^{n}$ 
this interpolated value, the left hand side in (10) is approximated by a finite difference so that a discrete formulation of Equation (6b) is

$$
\frac{h_{i}^{n+1}-\hat{h}_{i}^{n}}{\Delta t}=\frac{p_{*}^{\prime}\left(t^{n}\right)+\Phi\left(t^{n}, \xi_{i}^{n}\right)+\left[\partial_{y}\left(\Lambda\left(h^{n}, p_{*}\left(t^{n}\right)\right) \partial_{y} h^{n+1}\right)\right]_{y_{i}}}{\rho\left(\hat{h}_{i}^{n}, p_{*}\left(t^{n}\right)\right)} .
$$

The diffusion term is implicited but the conductivity is linearised as set to time $t^{n}$. It is discretised by means of a 3-point finite difference

$$
\begin{aligned}
{\left[\partial_{y}\left(\Lambda\left(h^{n}, p_{*}\left(t^{n}\right)\right) \partial_{y} h^{n+1}\right)\right]_{y_{i}} } & =\frac{\Lambda\left(h_{i+1 / 2}^{n}, p_{*}\left(t^{n}\right)\right)\left(\partial_{y} h^{n+1}\right)_{i+1 / 2}-\Lambda\left(h_{i-1 / 2}^{n}, p_{*}\left(t^{n}\right)\right)\left(\partial_{y} h^{n+1}\right)_{i-1 / 2}}{\Delta y} \\
& =\frac{\Lambda\left(h_{i+1 / 2}^{n}, p_{*}\left(t^{n}\right)\right)\left(h_{i+1}^{n+1}-h_{i}^{n+1}\right)}{\Delta y^{2}}-\frac{\Lambda\left(h_{i-1 / 2}^{n}, p_{*}\left(t^{n}\right)\right)\left(h_{i}^{n+1}-h_{i-1}^{n+1}\right)}{\Delta y^{2}} .
\end{aligned}
$$

The intermediate conductivity $\Lambda\left(h_{i+1 / 2}^{n}, p_{*}\left(t^{n}\right)\right)$ is computed as the harmonic mean of $\Lambda\left(h_{i}^{n}, p_{*}\left(t^{n}\right)\right)$ and $\Lambda\left(h_{i+1}^{n}, p_{*}\left(t^{n}\right)\right)$ as in [11].

Close to the boundary, a special treatment must be done as the characteristic curve arising from $\left(t^{n+1}, y_{i}\right)$ may cross the boundary between $t^{n}$ and $t^{n+1}$ (see [19] for more details).

The resulting implicit numerical scheme yields a tridiagonal linear system which can be solved without matrix inversion by means of the Thomas algorithm [1].

Although the scheme is unconditionnaly stable, a restriction upon the time step is imposed to ensure a good level of accuracy, either by a standard CFL condition $\Delta t \leq \Delta y / \max _{i}\left|v_{i}^{n}\right|$ or by a threshold.

The velocity field is recovered by integrating the divergence constraint (6a). But a particular attention must be paid in order to avoid instabilities due to the discontinuity of $\beta$ and $c^{2}$. In cells $\left(y_{i-1}, y_{i}\right)$ where phase transition occurs, i.e. when $\left(h_{\ell}^{s}-h_{i-1}^{n+1}\right)\left(h_{i}^{n+1}-h_{\ell}^{s}\right)>0$ or $\left(h_{q}^{s}-h_{i-1}^{n+1}\right)\left(h_{i}^{n+1}-h_{q}^{s}\right)>0,{ }^{8}$ the integral must be split into two parts: one over $\left(y_{i-1}, y_{*}^{n+1}\right)$ using liquid parameters and one over $\left(y_{*}^{n+1}, y_{i}\right)$ using mixture parameters, where $\left(y_{*}^{n+1}, h_{\ell}^{s}\right)$ is a linear approximation of the function passing through $\left(y_{i-1}, h_{i-1}^{n+1}\right)$ and $\left(y_{i}, h_{i}^{n+1}\right)$.

In the sequel, the adapted MOC scheme is called "MOC-d" scheme. We mention that when $\Lambda \equiv 0$, another scheme was designed in [3] to solve (10). Named INTMOC, it was proven to be convergent and more accurate for cases under interest. That is why the INTMOC scheme is used in the sequel for comparisons with the non-diffusive case.

\subsection{Simulations}

Unless specified, the data are set in Table 3 for the $1 \mathrm{D}$ numerical simulations. The enthalpy at the bottom $h_{e}$ is computed by inverting the EOS (3), namely

$$
h_{e}=q_{\ell}+\frac{\gamma_{\ell}}{\gamma_{\ell}-1} \frac{p_{*}+\pi_{\ell}}{\rho_{e}} \approx 1.191726 \times 10^{6} \mathrm{~J} \cdot \mathrm{K}^{-1} .
$$

\footnotetext{
${ }^{8}$ We do not present cases where pure vapour appears as we lack theoretical information. The algorithm is however implemented to handle all phases.
} 


\begin{tabular}{ccc}
\hline$L_{y}$ & Length of the core & $4.2 \mathrm{~m}$ \\
\hline$N_{y}$ & Number of spatial nodes & 100 \\
\hline$\Delta t$ & Time step & $0.01 \mathrm{~s}$ \\
\hline
\end{tabular}

(a) Discretisation parameters

\begin{tabular}{ccc}
\hline$p_{*}$ & Thermodynamic pressure & $155 \times 10^{5} \mathrm{~Pa}$ (constant) \\
\hline$\Phi$ & Power density & $170 \times 10^{6} \mathrm{~W} \cdot \mathrm{m}^{-3}$ (constant) \\
\hline$\rho_{e}$ & Input density & $750 \mathrm{~kg} \cdot \mathrm{m}^{-3}$ (constant) \\
\hline$v_{e}$ & Input velocity & $1 \mathrm{~m} \cdot \mathrm{s}^{-1}$ (constant) \\
\hline$h_{0}$ & Initial enthalpy & $h_{e}($ constant $)$ \\
\hline$v_{0}$ & Initial velocity & $v_{e}+\frac{\beta\left(h_{0}, p_{*}\right)}{p_{*}} \Phi y$ \\
\hline
\end{tabular}

(b) Physical parameters

TABLE 3. Parameters for 1D simulations

We recall that parameters of the Stiffened Gas law for this value of $p_{*}$ can be found in [3, Appendix B], as well as enthalpies at saturation $\left(h_{\ell}^{s}=1.627 \times 10^{6} \mathrm{~J} \cdot \mathrm{K}^{-1}\right.$ and $\left.h_{q}^{s}=3.004 \times 10^{6} \mathrm{~J} \cdot \mathrm{K}^{-1}\right)$.

The following simulations are not related to physical configurations (except $\S 2.3 .3$ and $\S 2.3 .5-\beta$ ) and are run in order to assess the modified algorithm and the model itself. Comparisons to the steady state from Prop. 1, compatibility with the non-diffusive case and assessment of the robustness of the algorithm are made. Cases 2.3.1 to 2.3.4 are set with $p_{*}$ constant in order to focus on the conductivity. The reader is reminded of the fact that only the Stiffened Gas EOS is used in the present paper as the extension to tabulated laws does not raise difficulties.

\subsubsection{Convergence for $\lambda \rightarrow 0$}

Let us first investigate the case of a constant conductivity $\lambda_{l}=\lambda_{q}=\lambda \rightarrow 0$. The purpose is to determine whether the model for non-zero small conductivities is close to the non-diffusive model studied in previous papers [2,3]. We compare the numerical results obtained by means of the MOC-d scheme to simulations of the LMNC-model for $\lambda=0$ by means of the INTMOC scheme. The $\mathrm{L}^{2}$-norm pictured on Fig. 1 shows that both solutions converge to the same limit as $\lambda$ goes to 0 .

\subsubsection{Influence of high conductivities}

Keeping a constant conductivity in both liquid and gas, we increase the corresponding value $\left(\lambda_{\ell}=\right.$ $\lambda_{g}=10^{6} \mathrm{~W} \cdot \mathrm{m}^{-1} \cdot \mathrm{K}^{-1}$ ) in order to highlight the discrepancy between non-diffusive and diffusive cases. Unlike the following example where both solutions match, we observe distinct numerical asymptotic solutions. In the mixture where $\Lambda=0$, both solutions are parallel straight lines. It is in the liquid phase that they have different shapes (Fig. 2(a)). 
We recover the steady solution from Prop. 1 which is an exponential-like curve. Figure 2(b) shows that the numerical solution converges to the steady solution. The $\mathrm{L}^{2}$-error is bounded from below by $10^{-2}$ which corresponds to the discretisation error $\left(\Delta y \approx 10^{-2}\right)$.

\subsubsection{Thermal conductivity in physical situations}

We then run the algorithm to a configuration with physical orders of magnitude for the thermal conductivity in liquid $\left(\lambda_{l}=0.5 \mathrm{~W} \cdot \mathrm{m}^{-1} \cdot \mathrm{K}^{-1}\right)$ and vapour $\left(\lambda_{g}=0.1 \mathrm{~W} \cdot \mathrm{m}^{-1} \cdot \mathrm{K}^{-1}\right)$. According to Fig. 1, the error with the non-diffusive case is of order $10^{-6}$ which is confirmed by next figures.

Figures 3 show the results at time $25 \mathrm{~s}$ where a numerical asymptotic state is reached. We deduce from Fig. 3(a) that this state is made of liquid and mixture (given that $h_{\ell}^{s}=1.627 \times 10^{6} \mathrm{~J} \cdot \mathrm{K}^{-1}$ ). The pointwise error is of order $10^{-4}$ and is larger in the mixture phase. This can be explained by the fact that $\Lambda$ vanishes in the mixture and the MOC-d scheme degenerates to the standard MOC scheme which turns out to be less accurate than the INTMOC scheme (see $[3, \S 5.1])$.

Hence the thermal conductivity does not play a major role for physical values which tends to legitimate not to take it into account in future works.

\subsubsection{Robustness}

We then consider a variable power density

$$
\Phi(t, y)= \begin{cases}600 \times 10^{6} \mathrm{~W} \cdot \mathrm{m}^{-3}, & \text { if } 0 \leq y \leq 1.6 \\ 0 \mathrm{~W} \cdot \mathrm{m}^{-3}, & \text { if } 1.6<y<1.8 \\ -400 \times 10^{6} \mathrm{~W} \cdot \mathrm{m}^{-3}, & \text { if } 1.8 \leq y \leq L_{y}\end{cases}
$$

to assess the robustness of the diffusive algorithm. Conductivities are set as in $\S 2.3 .3$. A positive power density makes the enthalpy increase (some mixture appears) while a negative one cools the flow. This is what the algorithm yields as seen on Figures 4. It manages to handle phase transition liquid to mixture (as previously) as well as mixture to liquid.

\subsubsection{Depressurisation processes}

As simulations tend to prove the low influence of the conductivity, we take $\lambda_{l}=\lambda_{g}=0$ and we focus on the effects of variable pressures. Two cases are considered: $\alpha$ concerns a toy-case (linear depressurisation to $40 \%$ to the initial pressure, Fig. 5(a)) to validate the algorithm whereas $\beta$ corresponds to physical data of a failure of the pressuriser (Fig. 5(b)).

In case $\alpha$, the depressurisation occurs once the asymptotic state is reached in initial conditions. Figures shows that the lower the thermodynamic pressure $p_{*}$, the lower the temperature at saturation $T^{s}$ which can be noticed as the plateau goes down as time goes on (Fig. 5(c)). Simultaneously, the rate of mixture increases (Fig. 5(e)) which can be accounted for by the fact that the enthalpy at saturation in the liquid phase $h_{\ell}^{s}$ decreases as $p_{*}$ does. The profile of the temperature in the liquid is the same as the steady state for $\lambda \equiv 0$ does not depend on data at saturation nor on the EOS (see [3, Rem. 4.5] and [6, Prop. 5.1]).

In case $\beta$, the depressurisation occurs at time $1 \mathrm{~s}$ while the asymptotic state is not yet reached (no mixture has appeared - Fig. 5(f)). That is why we observe the plateau going up then down (Fig. 5(d)). After some seconds, an asymptotic state is reached where most of the core is under mixture phase. 
As for the computational time, the variations of thermodynamic pressure require to compute data at saturation $\left(T^{s}, h_{\ell}^{s}, h_{q}^{s}\right)$ at each time step. When using the Stiffened Gas law, it has no impact compared

to the constant case. However, with tabulated EOS, each fitting polynomial of thermodynamic variables (density, temperature, compressibility coefficient, ...) must be recalculated at each time step. This would have a significant computational time but much less important than for classical compressible models where polynomials are also different at each space node.

\section{NumERICAL APPROACH IN DIMENSION 2}

\subsection{Weak formulation}

We now turn to the adaptation of the numerical scheme designed in [5] for $2 D$ flows when taking into account thermal diffusion and depressurisation processes. The original algorithm is a 1st order Lagrange finite element (FE) scheme coupled to the method of characteristics integrated in the FE software FreEFEM ++ . Due to the discontinuities of some variables involved in the model $\left(\Lambda, \beta, c^{2}\right.$, ... ), we decided to apply the Discontinuous Galerkin method also implemented in FreEFEM ++ . Wellsuited to this kind of solutions, the fact still remains it induces a significant rise of the computational time as unknowns are located on vertices and edges.

The domain is $\Omega_{2}=\left[0, L_{x}\right] \times\left[0, L_{y}\right]$ which is tesselated with triangles $\left(T_{k}\right)_{1 \leq k \leq K}$. The set of all edges is denoted by $\mathcal{E}_{K}$.

The numerical scheme is the one based on the weak formulation of the linearised model: "Find $(h, \boldsymbol{u}, \bar{p}) \in\left(h_{e}+\mathrm{H}\right) \times\left(\boldsymbol{u}_{e}+\mathrm{U}\right) \times \mathrm{H}^{1}$ such that for all $(\tilde{h}, \tilde{\boldsymbol{u}}, \tilde{p}) \in \mathrm{H} \times \mathrm{U} \times \mathrm{H}^{1}$

$$
\begin{gathered}
\iint_{\Omega_{2}} \tilde{p}(\nabla \cdot \boldsymbol{u}) \mathrm{d} \boldsymbol{x}=\iint_{\Omega_{2}}\left(\frac{\beta\left(h^{n}, p_{*}\left(t^{n}\right)\right) \Phi}{p_{*}\left(t^{n}\right)}-\frac{p_{*}^{\prime}\left(t^{n}\right)}{\rho\left(h^{n}, p_{*}\left(t^{n}\right)\right) c^{2}\left(h^{n}, p_{*}\left(t^{n}\right)\right)}\right) \tilde{p} \mathrm{~d} \boldsymbol{x} \\
-\iint_{\Omega_{2}} \frac{\Lambda\left(h^{n}, p_{*}\left(t^{n}\right)\right)}{p_{*}\left(t^{n}\right)} \nabla h^{n+1} \cdot\left[\beta\left(h^{n}, p_{*}\left(t^{n}\right)\right) \nabla \tilde{p}+\tilde{p} \frac{\partial \beta}{\partial h}\left(h^{n}, p_{*}\left(t^{n}\right)\right) \nabla h^{n}\right] \mathrm{d} \boldsymbol{x} \\
+\int_{\mathcal{E}_{K}} \llbracket \frac{\beta\left(h^{n}, p_{*}\left(t^{n}\right)\right) \Lambda\left(h^{n}, p_{*}\left(t^{n}\right)\right)}{p_{*}\left(t^{n}\right)}\left(\nabla h^{n+1} \cdot \boldsymbol{n}_{K}\right) \tilde{p} \rrbracket \mathrm{d} \varsigma, \\
\iint_{\Omega_{2}}\left(h^{n}, p_{*}\left(t^{n}\right)\right) \frac{h^{n+1}-h^{n}\left(\boldsymbol{\xi}^{n}\right)}{\Delta t} \tilde{h} \mathrm{~d} \boldsymbol{x}= \\
\iint_{\Omega_{2}}\left(\Phi+p_{*}^{\prime}\left(t^{n}\right)\right) \tilde{h} \mathrm{~d} \boldsymbol{x}-\iint_{\Omega_{2}} \Lambda\left(h^{n}, p_{*}\left(t^{n}\right)\right) \nabla h^{n+1} \cdot \nabla \tilde{h} \mathrm{~d} \boldsymbol{x} \\
+\int_{\mathcal{E}_{K}} \llbracket \Lambda\left(h^{n}, p_{*}\left(t^{n}\right)\right)\left(\nabla h^{n+1} \cdot \boldsymbol{n}_{K}\right) \tilde{h} \rrbracket \\
\mathrm{d} s
\end{gathered}
$$




\begin{tabular}{ccc}
\hline$p_{*}$ & Thermodynamic pressure & $155 \times 10^{5} \mathrm{~Pa}$ (constant) \\
\hline$\mu_{0}$ & Dynamic viscosity & $8.4 \times 10^{-5} \mathrm{~kg} \cdot \mathrm{m}^{-1} \cdot \mathrm{s}^{-1}$ (constant) \\
\hline$\Phi$ & Power density & $800 \Phi_{0} \exp \left(\frac{-1}{r^{2}-|\boldsymbol{x}|^{2}}\right) \mathbf{1}_{\{|\boldsymbol{x}| \leq r\}}$ \\
\hline$\rho_{e}$ & Input density & $750 \mathrm{~kg} \cdot \mathrm{m}^{-3}($ constant $)$ \\
\hline $\boldsymbol{u}_{e}$ & Input velocity & $\left(0, v_{e}\right)$ with $v_{e}=1.3 \mathrm{~m} \cdot \mathrm{s}^{-1}$ (constant) \\
\hline$h_{0}$ & Initial enthalpy & $h_{e}$ (constant) \\
\hline $\boldsymbol{u}_{0}$ & Initial velocity & $\left(0, v_{e}\right)$ (constant) \\
\hline
\end{tabular}

TABLE 4. Parameters for 2D simulations

$$
\begin{aligned}
\iint_{\Omega_{2}} \rho\left(h^{n}, p_{*}\left(t^{n}\right)\right) \frac{\boldsymbol{u}^{n+1}-\boldsymbol{u}^{n}\left(\boldsymbol{\xi}^{n}\right)}{\Delta t} \cdot \tilde{\boldsymbol{u}} \mathrm{d} \boldsymbol{x} & \\
-\frac{\mu_{0}}{2} \iint_{\Omega_{2}}\left(\nabla \boldsymbol{u}+(\nabla \boldsymbol{u})^{T}\right):: & \left(\nabla \tilde{\boldsymbol{u}}+(\nabla \tilde{\boldsymbol{u}})^{T}\right) \mathrm{d} \boldsymbol{x}+\eta_{0} \iint_{\Omega_{2}}(\nabla \cdot \boldsymbol{u})(\nabla \cdot \tilde{\boldsymbol{u}}) \mathrm{d} \boldsymbol{x} \\
& +\iint_{\Omega_{2}}(\nabla \cdot \tilde{\boldsymbol{u}})^{n+1} \mathrm{~d} \boldsymbol{x}+\iint_{\Omega_{2}} \rho\left(h^{n}, p_{*}\left(t^{n}\right)\right) \boldsymbol{g} \cdot \tilde{\boldsymbol{u}} \mathrm{d} \boldsymbol{x},^{\prime \prime}
\end{aligned}
$$

where

$$
\mathrm{H}=\left\{h \in \mathrm{H}^{1}\left(\Omega_{2}\right), h(x, 0)=0\right\} \text { and } \mathrm{U}=\left\{\boldsymbol{u} \in \mathrm{H}^{1}\left(\Omega_{2}\right)^{2}, \boldsymbol{u}(x, 0)=\mathbf{0}, u(0, y)=u\left(L_{x}, y\right)=0\right\} .
$$

Similarly to the $1 \mathrm{D}$ section - see (11), $\boldsymbol{\xi}^{n}$ denotes the foot of the characteristic curve, i.e. $\boldsymbol{\xi}^{n}(\boldsymbol{x})=$ $\chi\left(t^{n} ; t^{n+1}, \boldsymbol{x}\right)$ where $\boldsymbol{\chi}$ is the solution to the 2D counterpart of ODE (9).

Jumps involved in (12) account for potential discontinuities. Within pure phases solutions are expected to be smooth as shown in dimension 1 but jumps may occur with phase transition.

To improve the accuracy of the results, we refine the mesh (using the isoline package in FrEEFEM ++ ) in the mixture and vapour phases where corresponding domains can be small (bubbles).

\subsection{Numerical results}

We take $L_{x}=1 \mathrm{~m}$ and $L_{y}=3 \mathrm{~m}$. Other parameters are specified in Table 4 . In order to make vapour appear, we consider an overestimated ${ }^{9}$ power density that is compactly supported with a disk of radius $r=0.5$.

Due to strong variations of the velocity field when passing from one phase to another, a classical hyperbolic CFL condition for the time step is necessary rather than an arbitrary threshold. As stated before, to capture accurately the surfaces where phase change occurs, a refinement procedure is run in mixture and vapour phases. The results are analysed from two points of view: we focus first on the accuracy and the computational efficiency and then on the influence of the thermal conductivity to confirm preliminary results in $1 \mathrm{D}$.

\footnotetext{
${ }^{9}$ Compared to $\Phi_{0}$ in Table 3(b).
} 


\subsubsection{Accuracy issues and adaptative mesh refinement}

Due to the specifications of FrefFem ++ , mesh definitions are parametrised by the number of nodes on each boundary. For the accuracy investigation, we consider:

- A fine mesh corresponding to 100 nodes on horizontal boundaries (top and bottom) and 300 nodes on vertical boundaries (lateral walls);

- Coarser meshes based on a 25 horizontal / 75 vertical nodes with different rates of refinement in mixture and pure vapour phases (see the mesh on Figure 6 and legends of Figures 7-8). We mention that the mesh refinement procedure is adaptive: the mesh evolves in time as it depends on the appearance/disappearance of phases.

We can observe on the temperature representations (Fig. 7) the level sets $h=h_{\ell}^{s}$ and $h=h_{q}^{s}$ depicted with white lines separating phases. At the selected time $(0.59 \mathrm{~s})$ the three phases are present and we recover a constant temperature (equal to $T^{s}$ ) in the whole mixture domain. We also remark that the

finer the mesh, the wider the vapour domain. From a computational point of view, the finest refinement runs 5 -time faster than the finest mesh. Hence the refinement strategy turns out to be relevant in the balance accuracy/efficiency.

Figure 8 represents the mass fraction on the different meshes. We can observe the Rayleigh-Taylor instability due to the gravity field: the water is flowing upwards while the gravity is pointing downwards. The density decreases as the enthalpy increases which makes the mixture lighter than the liquid. The lighter phase is then sped up through the heavier phase above which induces the instability. We also notice that the fineness of the mesh influences neither the shape of the mixture nor the velocity at which the front evolves. The very difference lies in the domain occupied by vapour which is wider in the right case.

\subsubsection{Influence of the thermal conductivity}

We investigate here the influence of taking into account of the thermal conduction terms. We run the non-diffusive algorithm designed in [5] and the diffusive version described above (§3.1) over the same fine mesh with 100 horizontal / 300 vertical nodes. The computational times are almost the same. The conductivity is set to standard physical values $\left(\lambda_{\ell}=0.6 \mathrm{~W} \cdot \mathrm{m}^{-1} \cdot \mathrm{K}^{-1}\right.$ and $\left.\lambda_{\mathrm{g}}=0.08 \mathrm{~W} \cdot \mathrm{m}^{-1} \cdot \mathrm{K}^{-1}\right)$.

We see on Figure 9 that it is hard to distinguish the numerical solutions (enthalpy variable) for $\Lambda=0$ and $\Lambda \neq 0$. The cross-section in the middle of the core (on the right) shows that the only noticeable discrepancy occurs in the mixture phase. Hence these 2D results lead to the same conclusion as the previous 1D results: in nominal or accidental conditions in a PWR core, the thermal conductivity does not induce major changes in the solution of the LmNC-model. This legitimates not to take it into account in future works.

We finally mention that while a DG method was applied in the present paper to the standard formulation of the LMNC-model, another strategy was carried out in [6], namely an equivalent reformulation of the equations: the term between brackets in the right hand side of (1a) is replaced using Equation (1b) so that a standard Lagrange FE method can be applied. However, we do not state that any of these methods is proven to be more efficient. 


\section{Conclusion}

The present work consisted in taking into account additional physical phenomena in the modelling of fluid flows in nuclear reactor cores by means of the LMNC-model. On the one hand, thermal conduction effects induce a change in the mathematical structure of the equations and significant adaptations of the numerical schemes. But we managed to handle it in the 1D MOC scheme by solving directy the implicit version of the diffusive scheme and in the $2 \mathrm{D}$ scheme using features of the FreEFEM ++ software. We even improved the accuracy of the $2 \mathrm{D}$ algorithm by means of a refinement strategy. On the other hand, considering a (given) time-varying thermodynamic pressure does not raise particular issues from the computational point of view but may increase the computational time when fitting tabulated values for the equation of state.

The numerous simulations run in this paper suggest that the resulting numerical methods be robust to these modifications. They showed that the thermal conductivity does not play a major role in nominal or accidental conditions of a PWR core which enables to neglect it in future works. Thanks to the new algorithms we are now able to deal with more complex configurations like depressurisation processes for safety evaluations. The LMNC tools are improved step by step to provide reliable results for nuclear engineering. Perspectives will concern extensions to a 3D robust code and the coupling with neutronics to better model the power density $[7,8]$. Applications to other types of reactors may also be contemplated.

\section{ACKNOWLEDGEMENTS}

The project DiPLOMA on the occasion of the 2015 CEMRACS was funded by the CNRS project call "NEEDS" (nuclear, energy, environment, waste and society) for which CDMATH was selected in 2014 and 2015 .

We would also like to thank reviewers for fruitful comments.

\section{REFERENCES}

[1] W.F. Ames. Numerical methods for partial differential equations. Academic press, 1977.

[2] M. Bernard, S. Dellacherie, G. Faccanoni, B. Grec, O. Lafitte, T.-T. Nguyen, and Y. Penel. Study of low Mach nuclear core model for single-phase flow. In ESAIM:Proc, volume 38, pages 118-134, 2012.

[3] M. Bernard, S. Dellacherie, G. Faccanoni, B. Grec, and Y. Penel. Study of a low Mach nuclear core model for two-phase flows with phase transition I: stiffened gas law. Math. Model. Numer. Anal., 48(6):1639-1679, 2014.

[4] S. Dellacherie. On a low Mach nuclear core model. In ESAIM:Proc, volume 35, pages 79-106, 2012.

[5] S. Dellacherie, G. Faccanoni, B. Grec, E. Nayir, and Y. Penel. 2D numerical simulation of a low Mach nuclear core model with stiffened gas using FreeFem++. In ESAIM:ProcS, volume 45, pages 138-147, 2014.

[6] S. Dellacherie, G. Faccanoni, B. Grec, and Y. Penel. Study of a low Mach nuclear core model for two-phase flows with phase transition II: tabulated equation of state. 2016.

[7] S. Dellacherie, E. Jamelot, and O. Lafitte. A simple monodimensional model coupling an enthalpy transport equation and a neutron diffusion equation. Appl. Math. Lett., 62:35-41, 2016.

[8] S. Dellacherie and O. Lafitte. Une solution explicite monodimensionnelle d'un modèle simplifié de couplage stationnaire thermohydraulique-neutronique. Ann. Math. Québec, to appear.

[9] D. Di Pietro and A. Ern. Mathematical aspects of discontinuous Galerkin methods, volume 69. Springer Science \& Business Media, 2011.

[10] P. Embid. Well-Posedness of the nonlinear Equations for zero Mach Number Combustion. Communications in Partial Differential Equations, 12(11):1227-1283, 1987.

[11] R. Eymard, T. Gallouët, and R. Herbin. Finite volume methods, volume 7. 2000. 
[12] F. Gastaldi and A. Quarteroni. On the coupling of hyperbolic and parabolic systems: analytical and numerical approach. Appl. Numer. Math., 6(1):3-31, 1989.

[13] J.M. Gonzalez-Santalo and R.T. Lahey Jr. An exact solution for flow transients in two-phase systems by the method of characteristics. J. Heat Transf., 95(4):470-476, 1973.

[14] F. Hecht. New development in FreeFem++. J. Numer. Math., 20(3-4):251-266, 2012.

[15] S. Klainerman and A. Majda. Compressible and incompressible flows. Comm. Pure Appli. Math., 35:629-651, 1982.

[16] R. Klein. Semi-implicit extension of a Godunov-type scheme based on low Mach number asymptotics I: Onedimensional flow. J. Comput. Phys., 121(2):213-237, 1995.

[17] E.W. Lemmon, M.O. McLinden, and D.G. Friend. Thermophysical Properties of Fluid Systems. National Institute of Standards and Technology, Gaithersburg MD, 20899.

[18] A. Majda and J. Sethian. The derivation and numerical solution of the equations for zero Mach number combustion. Combust. Sci. Technol., 42(3-4):185-205, 1985.

[19] Y. Penel. An explicit stable numerical scheme for the 1d transport equation. Discrete Contin. Dyn. Syst. Ser. S, 5(3):641-656, 2012.

[20] Y. Penel, S. Dellacherie, and B. Després. Coupling strategies for compressible - low Mach number flows. Math. Models Methods Appl. Sci., 25(6):1045, 2015.

[21] O. Pironneau. On the transport-diffusion algorithm and its applications to the Navier-Stokes equations. Numer. Math., 38:309-332, 1982. 


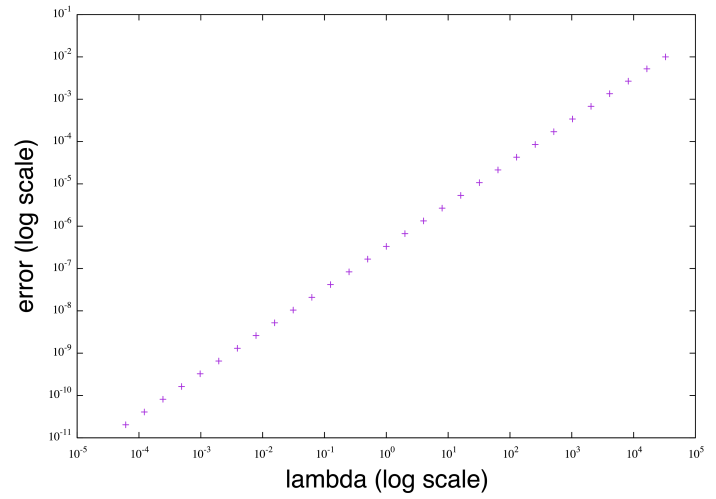

Figure 1. Case 2.3.1: Convergence for the enthalpy to the non-diffusive case in $\mathrm{L}^{2}$-norm

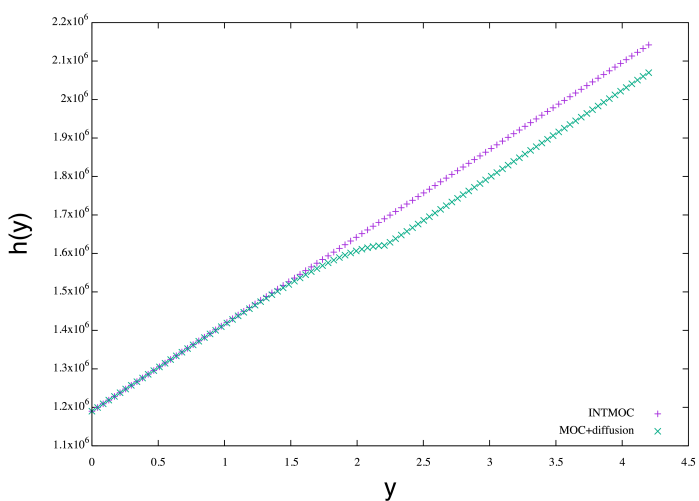

(a) Numerical solutions (enthalpy) at time $25 \mathrm{~s}$

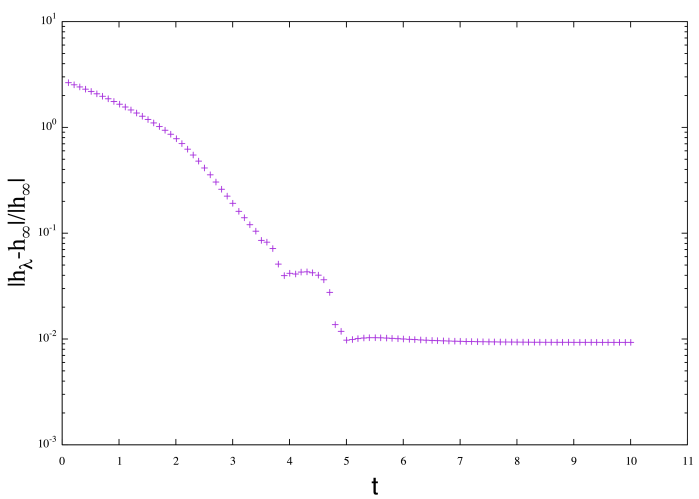

(b) Convergence to the steady state of Prop. 1

Figure 2. Case 2.3.2: Comparison between numerical solutions with and without thermal diffusion for unphysically high thermal conductivities when an asymptotic state is reached 


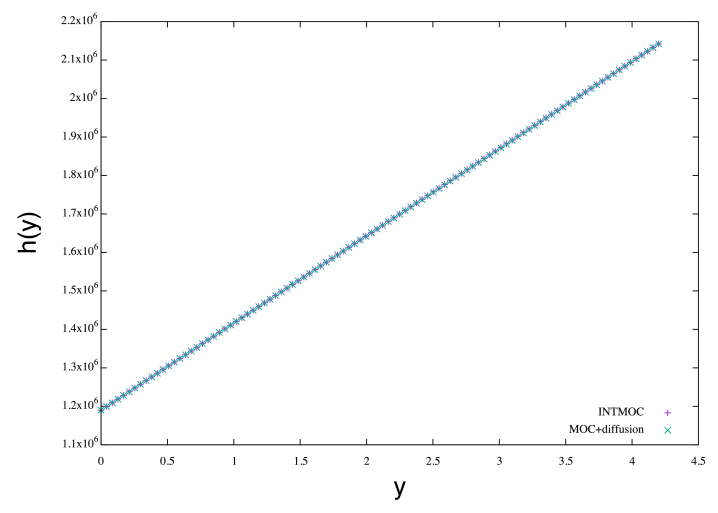

(a) Numerical solutions (enthalpy) at time $25 \mathrm{~s}$

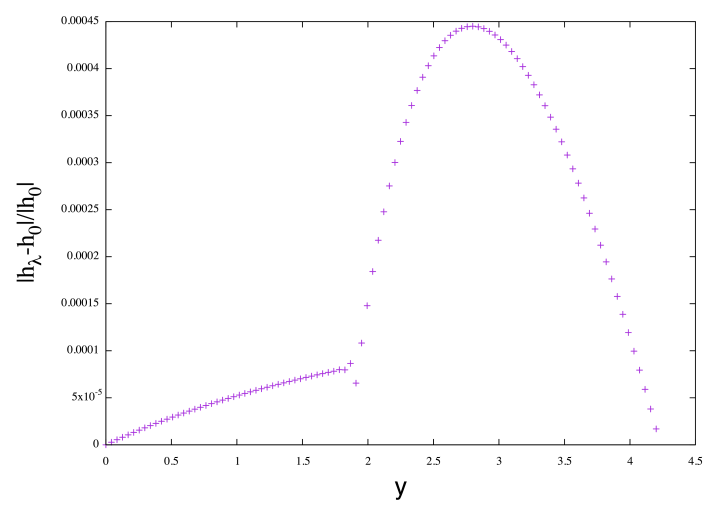

(b) Pointwise relative error (enthalpy) at time $25 \mathrm{~s}$

Figure 3. Case 2.3.3: Comparison between numerical solutions with and without thermal diffusion in standard conditions when an asymptotic state is reached

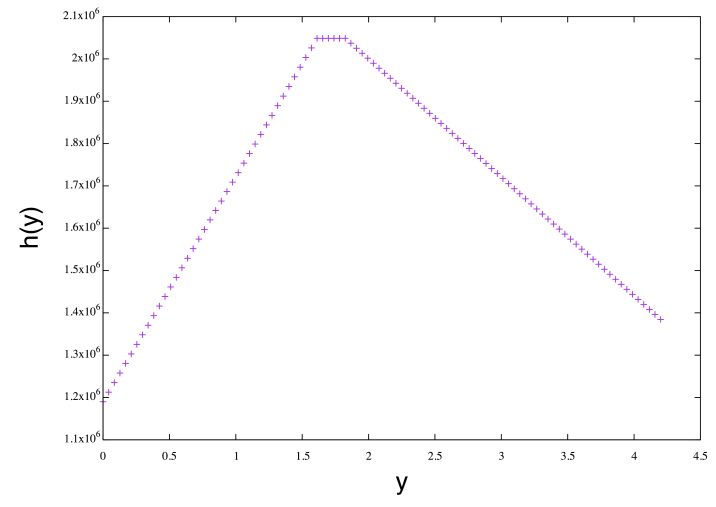

(a) Numerical enthalpy at time $25 \mathrm{~s}$

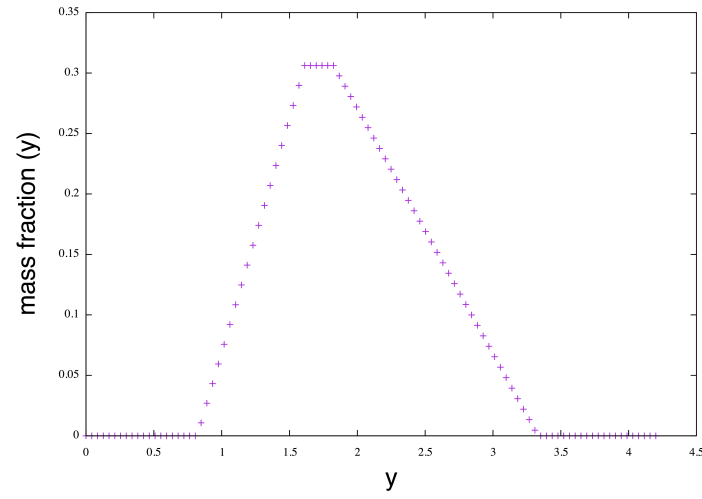

(b) Numerical mass fraction at time $25 \mathrm{~s}$

Figure 4. Case 2.3.4: Profile of the numerical solution for a piecewise constant power density 


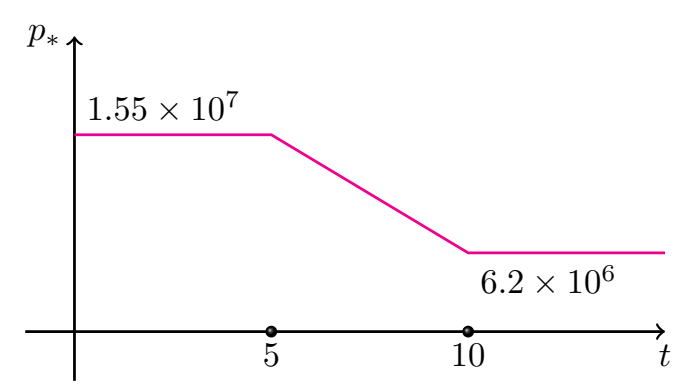

(a) Case $\alpha$ : profile of the depressurisation (linear decrease)

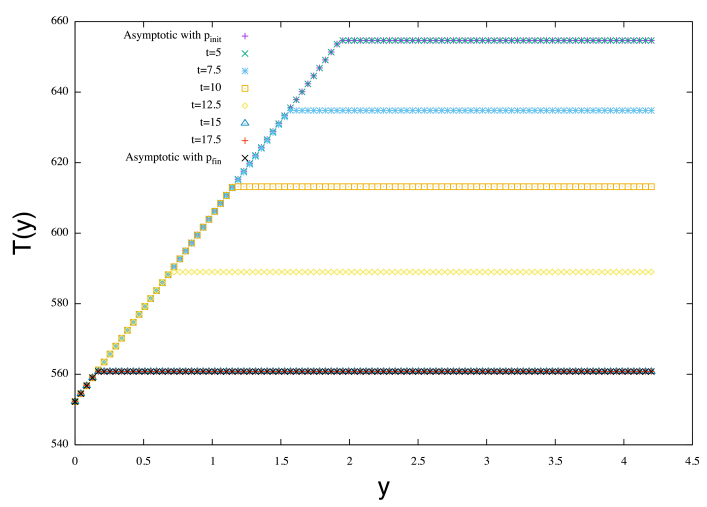

(c) Case $\alpha$ : Temperature at different times

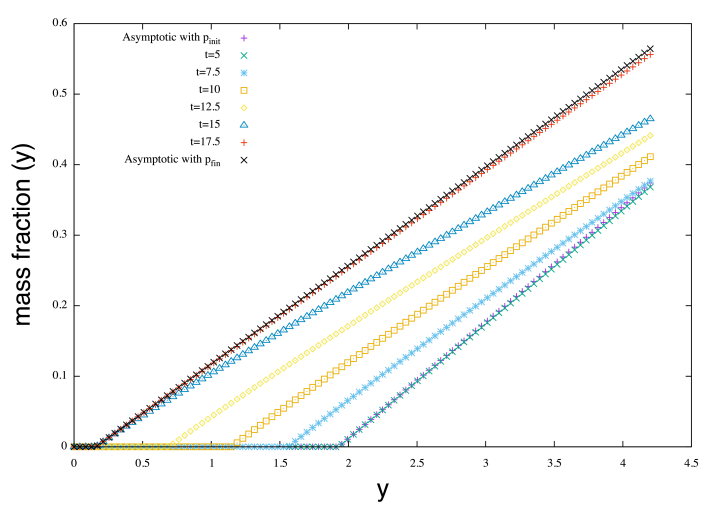

(e) Case $\alpha$ : Mass fraction at different times

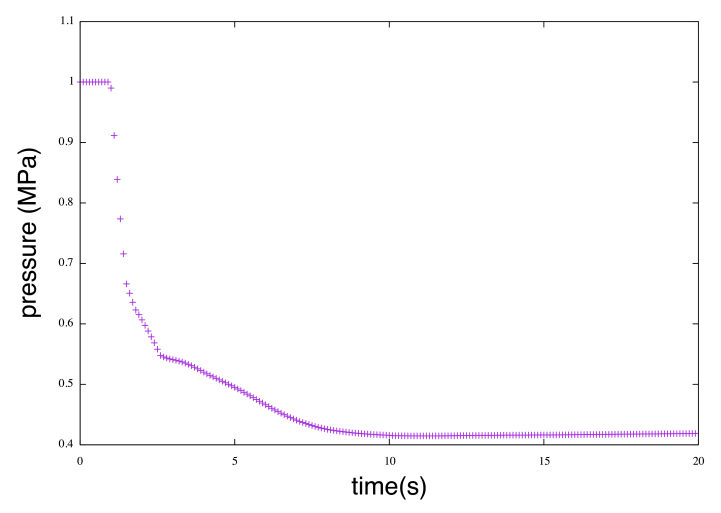

(b) Case $\beta$ : profile of the depressurisation (real case)

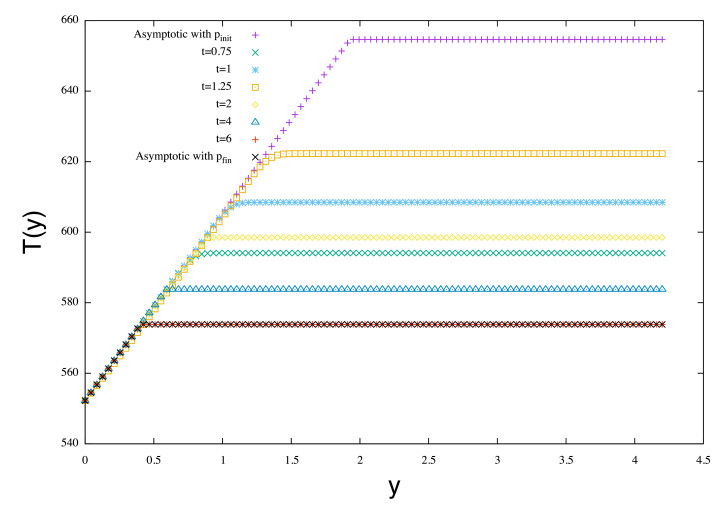

(d) Case $\beta$ : Temperature at different times

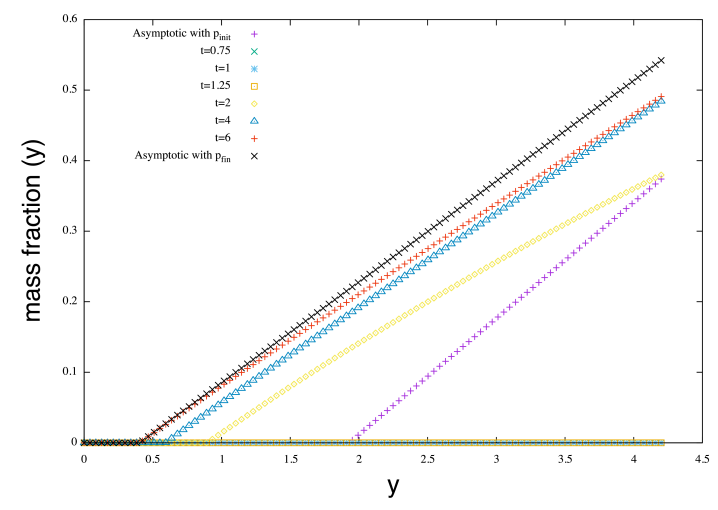

(f) Case $\beta$ : Mass fraction at different times

FiguRE 5. Case 2.3.5: Simulations of two depressurisation processes 


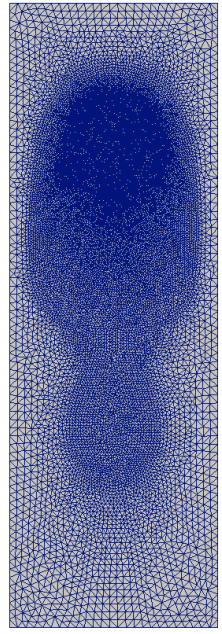

(a) Full mesh

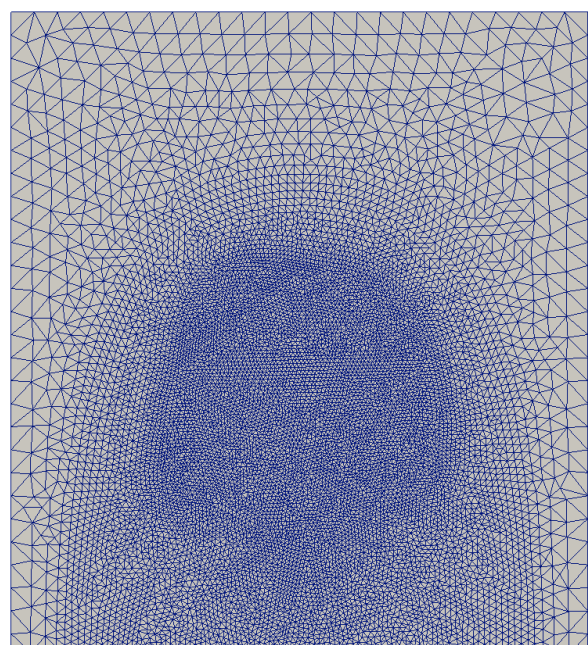

(b) Zoom around the domain occupied by vapour

Figure 6 . Mesh at time $t=0.59 \mathrm{~s}$ with 3 -time refinement in the mixture phase and 6 -time refinement in the pure vapour phase. Results obtained with this mesh are those on the far right of Figures 7 and 8.
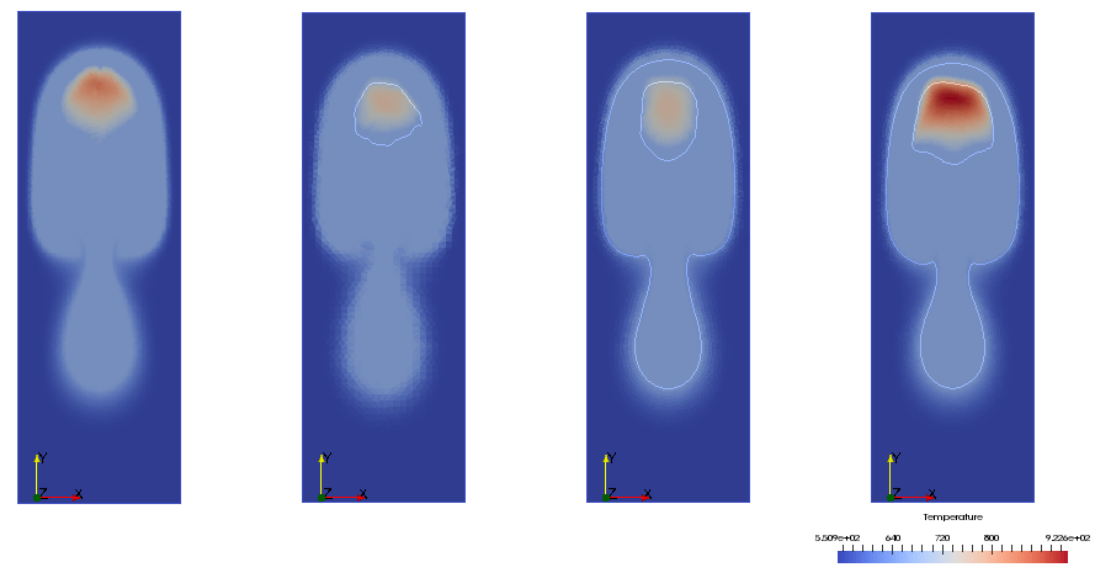

Figure 7 . Temperature at time $t=0.59 \mathrm{~s}$. From left to right: reference test case with a fine mesh $(100 \times 300) ; 25 \times 75$ with 4 -time refinement in the pure vapour phase; $25 \times 75$ with 2 -time refinement in the mixture phase and 4 -time refinement in the pure vapour phase; $25 \times 75$ with 3 -time refinement in the mixture phase and 6 -time refinement in the pure vapour phase (see also the mesh on Fig. 6 for this case). White lines correspond to the level sets of $h$ between phases. 

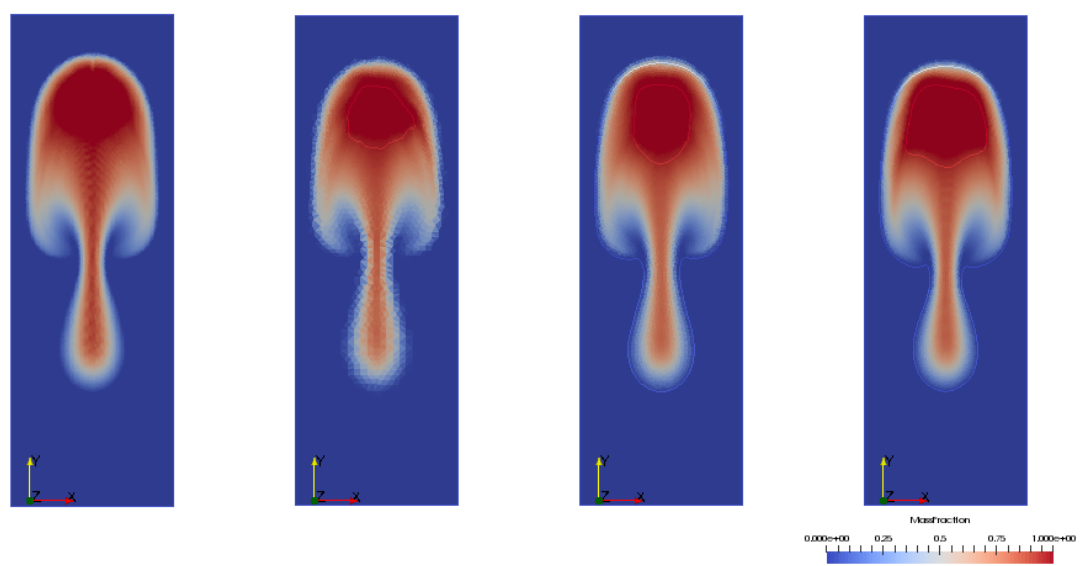

Figure 8. Mass fraction at time $t=0.59 \mathrm{~s}$. From left to right: reference test case with a fine mesh $(100 \times 300) ; 25 \times 75$ with 4 -time refinement in the pure vapour phase; $25 \times 75$ with 2 -time refinement in the mixture phase and 4 -time refinement in the pure vapour phase; $25 \times 75$ with 3 -time refinement in the mixture phase and 6 -time refinement in the pure vapour phase (see also the mesh on Fig. 6 for this case). White lines correspond to the level sets of $h$ between phases.
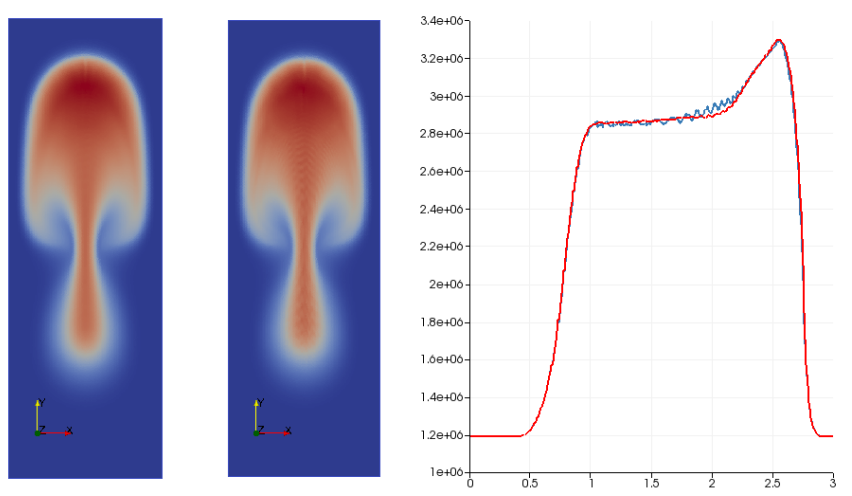

Figure 9 . Enthalpy at time $t=0.59 \mathrm{~s}$ on a fine mesh $(100 \times 300)$. Left: LmnCmodel with $\lambda=0$. Right: LMNC-model with $\lambda_{l}=0.6 \mathrm{~W} \cdot \mathrm{m}^{-1} \cdot \mathrm{K}^{-1}$ and $\lambda_{g}=$ $0.08 \mathrm{~W} \cdot \mathrm{m}^{-1} \cdot \mathrm{K}^{-1}$. The graph represents the values of the enthalpy on the vertical line $\left(x=L_{x} / 2,0 \leq y \leq L_{y}\right)$ : in red for the non-diffusive solution and in blue for the diffusive one. 